This is the author's version of the work. It is posted here by permission of the AAAS for personal use, not for redistribution. The definitive version was published in Science 347, 2015 Jan 2; DOI: 10.1126/science.1259859.

\title{
Mechanistic Insight from the Crystal Structure of Mitochondrial Complex I
}

Volker Zickermann ${ }^{1,2, *, \#}$, Christophe Wirth ${ }^{3, *}$, Hamid Nasiri ${ }^{4,5}$, Karin Siegmund ${ }^{1}$, Harald Schwalbe ${ }^{2,5}$, Carola Hunte ${ }^{3, \#}$, Ulrich Brandt ${ }^{2,6, \#}$

${ }^{1}$ Structural Bioenergetics Group, Institute of Biochemistry II, Medical School, GoetheUniversity, Frankfurt am Main, Germany

${ }^{2}$ Cluster of Excellence Frankfurt "Macromolecular Complexes", Goethe-University, Germany

${ }^{3}$ Institute for Biochemistry and Molecular Biology, ZBMZ, BIOSS Centre for Biological Signalling Studies, University of Freiburg, Germany

${ }^{4}$ Department of Chemistry, The University of Cambridge, Lensfield Road, Cambridge, United Kingdom

${ }^{5}$ Institute of Organic Chemistry and Chemical Biology, Center for Biomolecular Magnetic Resonance, Germany

${ }^{6}$ Nijmegen Center for Mitochondrial Disorders, Radboud University Medical Center, Nijmegen, The Netherlands

* These authors have contributed equally to this work.

\# To whom correspondence should be addressed:

Volker Zickermann, Institute of Biochemistry II, Medical School, Goethe-University, Max-vonLaue Str. 9, 60438 Frankfurt, Germany

Carola Hunte, Institute for Biochemistry and Molecular Biology, ZBMZ, BIOSS Centre for Biological Signalling Studies, University of Freiburg, Stefan Meier Str. 17, 79104 Freiburg, Germany

Ulrich Brandt, Nijmegen Center for Mitochondrial Disorders, Radboud University Medical Center, Geert-Grooteplein 10, Route 772, 6500 GA Nijmegen, The Netherlands 


\begin{abstract}
:
Proton-pumping complex I of the mitochondrial respiratory chain is among the largest and most complex membrane protein complexes. The enzyme contributes substantially to oxidative energy-conversion in eukaryotic cells. Its malfunctions are implicated in many hereditary and degenerative disorders. Here, we report the X-ray structure of mitochondrial complex I at 3.6$3.9 \AA$ resolution describing in detail the central subunits that execute the bioenergetic function. A continuous axis of basic and acidic residues running centrally through the membrane arm connects the ubiquinone reduction site in the hydrophilic arm to four putative proton-pumping units. The binding position for a substrate analogous inhibitor and blockage of the predicted ubiquinone binding site provide a model for the 'deactive' form of the enzyme. The proposed transition into the active form is based on a concerted structural rearrangement at the ubiquinone reduction site rendering support for a two-state stabilization-change mechanism of protonpumping.
\end{abstract}

\title{
One Sentence Summary:
}

The X-ray structure of proton-pumping mitochondrial complex I gives insight into the mechanism of energy-conversion. 
With a molecular mass of $\sim 1 \mathrm{MDa}$, proton-pumping NADH:ubiquinone oxidoreductase (complex I) is the largest membrane protein complex of the mitochondrial respiratory chain $(1$, 2). Complex I couples electron-transfer from NADH to ubiquinone to the translocation of four protons from the mitochondrial matrix to the intermembrane space (IMS) contributing greatly to the proton-motive force for ATP synthesis. Dysfunction of the enzyme is the most frequent cause of mitochondrial disorders (3) and has been implicated in numerous neurodegenerative conditions (4). Complex I is also a major source of deleterious reactive oxygen species (5). Mitochondrial complex I consists of 14 central subunits and a large number of accessory subunits. Specific inhibitors have been essential for unravelling structure/function relationships within complex I and are a focus of biomedical research, as some can induce Parkinson's disease (6). The central subunits of complex I harboring the bioenergetic core functions are conserved from bacteria to humans. Complex I from bacteria and from mitochondria of Yarrowia lipolytica, a yeast-genetic model to study eukaryotic complex I (7), were analyzed previously by X-ray crystallography (810). Yet, the catalytic mechanism has remained enigmatic. We here present the X-ray structure of all central subunits and the largest accessory subunit of mitochondrial complex I from $Y$. lipolytica providing insight into the molecular basis of redox-driven proton-pumping.

\section{Overall structure}

Complex I was purified and crystallized as previously described (8). Optimized cryoprotection and data collection improved X-ray diffraction in the best direction to $3.6 \AA$. SIRAS phases were obtained from highly redundant anomalous data from heavy-atom derivatives, FeSclusters and selenomethionine. Phases were combined, averaged and extended to the resolution of the native data set collected at $5 \mathrm{~K}$. Model building with the $3.8 \AA$ experimental electron-density map was aided by the large content of $\alpha$-helices, close to 100 selenomethionine positions and 
homology models based on the bacterial complexes $(10,11)$. Secondary structure constraints were applied for refinement. Anisotropy correction counteracted the lower order of the crystals in the membrane plane and the structure was refined at $3.9 \AA \times 3.9 \AA \times 3.6 \AA$ (Table S1, Fig. S1). The structure contains the majority of residues for central subunits and the largest accessory subunit (Table S2). Side-chains are well resolved in tightly packed subunits with high helix content and assignment of residues for these subunits ranges between 50 and $89 \%$. The end of the peripheral hydrophilic domain is less ordered (Fig. S1, Table S1).

Mitochondrial complex I has a slightly opened L-shape with an angle of $\sim 120^{\circ}$ between the two arms (Fig. 1). The matrix arm protrudes into the organelle interior and is oriented perpendicular to the membrane plane and the membrane arm. The 14 central subunits forming the core of the complex are surrounded by the accessory subunits comprising $44 \%$ of the total mass. Complex I is $250 \AA$ long and $190 \AA$ high with a width of $70 \AA$ for the membrane arm and $120 \AA$ for the matrix arm. The complex is organized in four functional modules $(1,8)$. The distal half of the matrix arm comprising the central $75-\mathrm{kDa}^{1}, 51-\mathrm{kDa}$ and $24-\mathrm{kDa}$ subunits forms the $\mathrm{N}$-module that oxidizes NADH. The proximal half comprising the central 49-kDa, 30-kDa, PSST and TYKY subunits is the Q-module that reduces ubiquinone and docks onto the membrane arm. A chain of eight canonical FeS-clusters (2) runs over a distance of $\sim 100 \AA$ through the matrix arm (Fig. 1C). The edge-to-edge-distances for seven of these clusters are $<14 \AA$ allowing fast electron-tunnelling (12). This arrangement establishes an electron-transfer path from the $[4 \mathrm{Fe}-$ 4S]-cluster of the 51-kDa subunit (cluster N3) to the [4Fe-4S]-cluster of PSST (cluster N2). Cluster N2 reduces ubiquinone and resides $\sim 30 \AA$ above the membrane plane (8). The slightly bent membrane arm features 82 transmembrane helices (TMH), with 64 of them contributed by central subunits (Fig. 2A). The proximal pump module $\left(\mathrm{P}_{\mathrm{P}}\right)$ comprises central subunits ND1,

\footnotetext{
${ }^{1}$ nomenclature as for bovine complex I is used throughout the text
} 
ND2, ND3, ND4L and ND6, while the distal pump module $\left(\mathrm{P}_{\mathrm{D}}\right)$ contains central subunits ND4 and ND5 (Fig. 2A).

\section{Membrane arm and proton-pumping modules}

The largest membrane-embedded subunits ND5, ND4 and ND2 (Fig. 2A) share a structurally highly similar core of 14 TMH (Fig. S2) with two repeats of five TMH (A: TMH4-8, B: TMH913) in inverted topology (Fig. S2). Each repeat features a discontinuous helix (TMH7a/b, TMH12a/b). Such helices are hallmarks of ion-translocating membrane proteins (13-15). Indeed, ND5, ND4 and ND2 are homologous to the Mrp Na $/ \mathrm{H}^{+}$antiporter family suggesting a role in proton-pumping $(8,11,16)$. ND5 has a C-terminal extension with a $>60-\AA$ long lateral helix lining ND5, ND4 and ND2 on the concave side of the arm close to the matrix-side (Fig. 2A), thus bridging the $\mathrm{P}_{\mathrm{P}}$ and $\mathrm{P}_{\mathrm{D}}$ modules. The very C-terminus of ND5 is anchored to ND2 via a V-shaped arrangement of TMH16 and TMH17. The lateral helix was previously identified in the low resolution analysis of mitochondrial complex I from $Y$. lipolytica (8) and is also present in the bacterial $(9,11)$ and bovine complexes $(17)$, in which its C-terminus is anchored by one TMH only. Whether this prominent structural element is involved energy-transduction for protonpumping as proposed previously $(8,9)$ remains controversial $(2)$.

Adjacent to ND2 are the three small central subunits ND4L, ND6, and ND3 that form two layers of straight helices crossing the membrane domain (Fig. 2A, Fig. S3A). Notably, TMH3 ${ }^{\text {ND6 }}$ features a $\pi$-bulge near the membrane center. Remote with respect to TMH1-3 ${ }^{\mathrm{ND} 6}$, TMH4 ${ }^{\mathrm{ND} 6}$ is laterally associated with the membrane arm and lines $\mathrm{TMH} 16^{\mathrm{ND}}$. On the intermembrane space side, a long loop links THM ${ }^{\mathrm{ND} 6}$ to a short surface helix $\left(\alpha_{4-5}\right)$ connecting to TMH5 ${ }^{\mathrm{ND} 6}$, which is

surrounded by helices of ND2, ND4L and ND3. In the bacterial complex $(9,11), \mathrm{TMH}^{\mathrm{ND} 6}$ sits closer to the core helices of ND6 at a position occupied in the mitochondrial complex by a single 
helix of an accessory subunit, tentatively identified as NUJM (17). TMH1 ${ }^{\text {ND3 }}$ is anchored remotely in a surface cleft of ND1. ND1 at the proximal end of the membrane arm comprises eight TMH and noticeable loop regions including short surface helices (Fig. 2A, Fig. S3B). The fold of TMH2-6 is similar to one repeat of the antiporter-like subunits. This agrees with homology predictions (18) and was noted in the bacterial complex (10). Notably, TMH5 is discontinuous in mitochondrial complex I.

The interior of the membrane arm is rich in polar and protonable residues constituting a unique hydrophilic central axis across all subunits (Fig. 2B). Towards the matrix and IMS, each of the antiporter-like subunits has typical arrays of titratable and polar residues constituting possible proton-uptake and -release pathways (Fig 2B, Fig S4). For ND5 (Fig. 2B), D397 at the membrane center is the first in a series of polar residues (Y392, E401, T403, S402) arranged along TMH12b to the IMS. Neighboring K396 connects this path to the central polar network with major contributions of protonable residues of TMH11 (H331, H335, K339). The likely entry-point to this network is $\mathrm{H} 251$ of $\mathrm{TMH} 8$, to which protons could be provided from the matrix along the tip of TMH7b, TMH8 and TMH10 (E241, S247, T244, K302). At the center of discontinuous TMH7, K226 (TMH7a) is sandwiched between the indole side-chains of W143 and W235 and connects on one side to H251 (involving S227, T254) and on the other side to TMH12 $2^{\text {ND4 }}$ via protonable residues of TMH5 and TMH6 (E144, R175, D178). A similar architecture is repeated in ND4 and ND2 as detailed in Fig. S4. Whereas the central network and the proposed proton-exit routes are consistent with suggestions for the bacterial complex, the proposed routes for proton-entry differ as they were assigned between TMH5 and TMH7 in bacteria $(10,19)$.

In summary, all three antiporter-like subunits carry the structural signature of a protonpumping unit with two structurally distinct discontinuous helices. Consistently, TMH7a/b holds a 
central lysine residue next to a glutamate of TMH5, and TMH12a/b is part of a central polar network connecting the proton-exit route along TMH12b with the likely proton-entry-point at loops TMH7b-8 and TMH9-10 (Fig. 2B). The dipoles of the discontinuous helices add to the polarity of the central axis, since TMH7a and 12a point with their C-termini to the center thereby introducing evenly spaced negative partial charges along the membrane arm. In contrast, the positive polarity of the $\mathrm{N}$-termini of $\mathrm{TMH} 7 \mathrm{~b}$ and $12 \mathrm{~b}$ is directed away from the central axis towards the periphery of the membrane arm (Fig. 2B,C).

In line with a pump-stoichiometry of $4 \mathrm{H}^{+} / 2 \mathrm{e}^{-}(20)$, a fourth potential proton-translocationpathway could be anticipated in the small NDs or ND1. From the neighboring residues E30 ND4L, E66 ${ }^{\mathrm{ND} 4 \mathrm{~L}}$ and $\mathrm{E} 131^{\mathrm{ND} 2}$ continuing the central axis, a row of polar residues towards the IMS at the interface of ND2 and ND4L could serve as proton-exit (Fig. 2B, Fig. S4). Directly above, a series of polar residues constitutes a potential fourth proton-entry at the interface initially also proposed to be present in bacterial complex I (19). The central axis extends further towards E196 at the center of discontinuous TMH5 $5^{\mathrm{ND} 1}$ along D67 $7^{\mathrm{ND} 3}, \mathrm{E} 69^{\mathrm{ND} 3}$, and E147 $7^{\mathrm{ND} 1}$. The $\pi$-bulge of TMH3 ${ }^{\mathrm{ND} 6}$ provides an additional polar contribution. From E196 ${ }^{\mathrm{ND} 1}$ onwards, a row of acidic residues of ND1 (D203, E206, E208, E210) continues upward towards the peripheral arm. A similar arrangement was described in complex I from T. thermophilus (10) and was interpreted as possible fourth pathway for proton-uptake replacing the one proposed earlier (19). However, based on the structure of mitochondrial complex I, the central axis of protonable residues is more likely to play a critical role in energy-transmission.

\section{Peripheral arm}

The peripheral arm comprises the $\mathrm{N}$ module extending into the matrix and the $\mathrm{Q}$ module docking it onto the membrane arm. The central subunits of the Q-module, 49-kDa, PSST, TYKY 
and $30-\mathrm{kDa}$ subunits, are related to $\mathrm{Ni}-\mathrm{Fe}$ hydrogenases $(1,21)$. The $49-\mathrm{kDa}$ subunit comprises two 3-stranded antiparallel $\beta$-sheets and several $\alpha$-helices. The longest helices form a prominent four-helix bundle inclining towards the membrane surface (Fig. 1, 3). PSST on the other side of the Q module contains a central 4-stranded parallel $\beta$-sheet surrounded by five helices, and harbors [4Fe-4S]-cluster $\mathrm{N} 2$. The $\mathrm{N}$-terminal helix $\alpha_{1}{ }^{\text {PSST }}$ protrudes towards the membrane surface (Fig. 1, Fig. 3B). TYKY provides contact to the N-module. Its ferredoxin-type fold holds two [4Fe-4S]-clusters (Fig. 3). The N-terminal helix $\alpha_{1}$ docks onto ND1 and is in contact to the 4-helix bundle of the 49-kDa subunit. The latter is also the docking-site for the $30-\mathrm{kDa}$ subunit, with a central 5-stranded $\beta$-sheet laterally surrounded by four $\alpha$-helices. In the N-module, the 75$\mathrm{kDa}$ subunit resides on the same side as PSST and coordinates one [2Fe-2S]- and two [4Fe-4S]clusters (Fig. 1, Fig. 3B). The 51-kDa subunit located above the 49-kDa subunit holds one [4Fe4S]-cluster and contains the binding sites for FMN and NADH in a Rossman-fold domain. The $24-\mathrm{kDa}$ subunit coordinates the $[2 \mathrm{Fe}-2 \mathrm{~S}]$-cluster detached from the electron-transfer chain leading to cluster N2. As noted previously (8), the Q module is rotated outwards about three degrees relative to the $\mathrm{N}$ module as compared to bacterial complex I (10).

The largest accessory subunit NUEM, an ortholog of the mammalian 39-kDa subunit, flanks the Q module and docks onto PSST (Fig. S5). It belongs to the large family of short-chain dehydrogenases (SDRs). A characteristic Rossman-fold typically found in all SDRs (22) is present in the $\mathrm{N}$-terminal domain with a central seven-stranded $\beta$-sheet surrounded by five $\alpha$ helices (Fig. 3; Fig. S5). NADPH binding to Y. lipolytica complex I was shown experimentally (23). The helices of the C-terminal domain characteristic for the subfamily of extended SDRs are in contact with the membrane arm, in line with the reported conformation specific cross-linking to ND3 (24). Assembly defects of $Y$. lipolytica complex I in deletion mutants (23) suggest a structural role, but the function of NUEM remains elusive. Some additional accessory subunits 
were provisionally localized in the Y. lipolytica complex I structure (Fig. S6) based on their tentative assignment in? the electron microscopic model of bovine complex I published during revision of this manuscript (17).

\section{Interface between peripheral and membrane arm and ubiquinone access}

Structural elements of the ND1, ND3, 49-kDa, PSST, and TYKY subunits form the interface between membrane and peripheral arm (Fig. 3). The matrix-surface of ND1 provides the main contact-area with the 49-kDa subunit and PSST. Helix $\alpha_{1}{ }^{\text {PSST }}$ touches surface helix $\alpha_{1-2}{ }^{\text {ND1 }}$. On the opposite side, helix $\alpha_{1}{ }^{\text {TYKY }}$ protrudes into a groove between the 4-helix bundle of the 49-kDa subunit and ND1. TMH1 ${ }^{\mathrm{ND} 3}$ and $\mathrm{TMH} 2^{\mathrm{ND} 3}$ are in contact with loop $\mathrm{TMH} 1-2^{\mathrm{ND} 1}$ including helix $\alpha_{1-2}{ }^{\mathrm{ND} 1}$ and with loop $\beta_{2}-\beta_{3}$ of the N-terminal $\beta$-sheet of the $49-\mathrm{kDa}$ subunit, respectively. At a central point of the interface, contact between the two arms of complex I involves loop TMH5$6^{\mathrm{ND} 1}\left(\mathrm{E} 206^{\mathrm{ND} 1}\right.$ to $\mathrm{G} 221^{\mathrm{ND} 1}$ ) (Fig. 5). This loop is rich in acidic residues, protrudes into a cleft between PSST and 49-kDa subunit and is in contact with loop $\beta 1-\beta 2\left(\mathrm{P} 89^{49-k D a}\right.$ to $\left.L 98^{49-k D a}\right)$ of the N-terminal $\beta$-sheet, where also loop TMH1-2 ${ }^{\mathrm{ND} 3}$ approaches.

Since cluster N2 resides well above the membrane plane, an access-path allowing the headgroup of the hydrophobic substrate ubiquinone to reach its electron-donor is needed (25). This path is provided by a quinone exchange-cavity crossing the interface region. Its opening lies between TMH1, TMH6 and amphipathic helix $\alpha_{1-2}{ }^{\mathrm{ND} 1}$ positioned at the periphery of the matrix bilayer leaflet. The cavity extends about $30 \AA$ towards the tip of loop $\beta 1-\beta 2^{49-k D a}$ (Fig. 4). The small triangular-shaped entry-pore is about $7 \AA$ wide and has a hydrophobic surface. The sidechain of A54 of $\alpha_{1-2}{ }^{\mathrm{ND} 1}$ points into the pore-opening. Substitution of the corresponding A52 ${ }^{\mathrm{ND} 1}$ in humans with threonine is among the most prevalent mutations leading to Leber's hereditary optic neuropathy and interferes with ubiquinone reduction (26). Furthermore, substitution of W77 ${ }^{\text {PSST }}$ 
lining the inner side of the narrow entry-passage with glutamate abolishes ubiquinone reductase activity (27). The surface and immediate vicinity of the cavity exhibits a bipartite distribution of charged residues. Towards the surface of the complex basic residues are present at the C-terminal end of $\mathrm{TMH}^{\mathrm{ND1}}(\mathrm{R} 27, \mathrm{~K} 28, \mathrm{R} 36, \mathrm{R} 37), \mathrm{TMH} 7^{\mathrm{ND} 1}$ and loop TMH7-8 ${ }^{\mathrm{ND} 1}$, some of which may interact with phospholipids. Deeper into the pocket towards the protein interior, the cavity is lined by acidic residues of TMH5 ${ }^{\mathrm{ND} 1}$ and adjacent loop TMH5-6 ${ }^{\mathrm{ND} 1}$ (D203, E206, E208, E210) (Fig. 4).

\section{Ubiquinone and inhibitor binding-sites}

To define the substrate- and inhibitor binding-sites within the Q-module, we co-crystallized complex I with brominated derivatives of 2-decyl-4-quinazolinylamine (DQA; Fig. 5). DQA is a potent class I/A type inhibitor competitive to piericidin A (28) that was shown to qualify as a true ubiquinone analogoue (29). Brominating the quinazoline scaffold (QA-1) increased the $\mathrm{IC}_{50}$ from $17 \mathrm{nM}$ for DQA to $320 \mathrm{nM}$ and replacing the side-chain with a brominated phenylethylamine moiety (QA-2) hardly affected binding ( $\mathrm{IC}_{50}=23 \mathrm{nM}$ ) (Fig S7). Anomalous Fourier electrondensity maps (Fig. 5) positioned the bromine atoms close to H95 $5^{49-k D a}$ at the deepest point of the quinone exchange-cavity (Fig. 5B). For both derivatives the geometrical constraints imposed by anomalous signals and structure are consistent with an identical toxophore position; a quinazoline ring was modelled into the structure to visualize its likely position. The planar aromatic ringsystem stacks between the tip of loop $\beta_{1}-\beta_{2}{ }^{49-k D a}$ and M91 ${ }^{\text {PSST }}$ (Fig. 5B) placing the bromine at the quinazoline ring and the one in the tail moiety $14 \AA$ and $16 \AA$ away from cluster N2, respectively. Supporting the notion that the position of the substrate analogs also reflects a

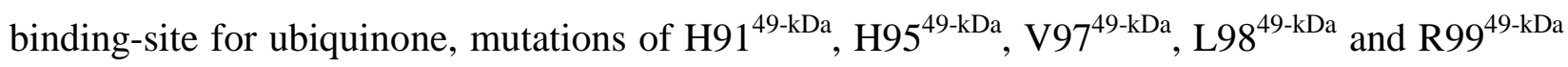
in loop $\beta_{1}-\beta_{2}{ }^{49-k D a}$ as well as substitution of M91 ${ }^{\text {PSST }}$ by lysine or glutamate cause drastic 
reduction of enzymatic activity $(21,27,30,31)$, while $\mathrm{M} 91 \mathrm{C}^{\mathrm{PSST}}$ showed a marked resistance against DQA and rotenone. Further residues (S192 ${ }^{49-\mathrm{kDa}}, \mathrm{M} 188^{49-\mathrm{kDa}}, \mathrm{F} 203^{49-\mathrm{kDa}}$ and $\mathrm{V} 88^{\mathrm{PSST}}$ ) with a role in ubiquinone or inhibitor binding (27) are located nearby (Fig 5).

Notably, some distinct structural features in mitochondrial complex I can explain a known functional difference to the bacterial enzyme: the quinone exchange-cavity does not provide access to several other residues (D143 ${ }^{49-\mathrm{kDa}}, \mathrm{Y} 144^{49-\mathrm{kDa}}, \mathrm{V} 145^{49-\mathrm{kDa}}, \mathrm{D} 458^{49-\mathrm{kDa}}, \mathrm{V} 460^{49-\mathrm{kDa}}$ ) for which biochemical evidence obtained with $Y$. lipolytica suggested a direct interaction with ubiquinone and inhibitors $(29,30)$. Indeed, binding of ubiquinone and piericidin A next to these residues and closer to cluster N2 was modelled into the structure of the bacterial enzyme (10) revealing a marked difference (Fig. S8) between the otherwise similar (Table S3) core-structures of $Y$. lipolytica and T. thermophilus complex I. Evidently, in the $Y$. lipolytica structure, access deeper into the protein is blocked by the tip of loop $\beta_{1}-\beta_{2}{ }^{49-\mathrm{kDa}}$ bringing $\mathrm{H} 95^{49-\mathrm{kDa}}$ close to $\mathrm{Y} 144^{49-}$ $\mathrm{kDa}$, while in the bacterial enzyme the ubiquinone head-group was located between these two residues (10). The different position of loop $\beta_{1}-\beta_{2}{ }^{49-\mathrm{kDa}}$ is accompanied by significantly different orientations of the adjacent acidic loop TMH5-6 $6^{\mathrm{ND} 1}$ and loop TMH1-2 ${ }^{\mathrm{ND} 3}$.

This observation provides a straightforward explanation for the reversible A/D-transition (32) occurring only in complex I of some eukaryotes including $Y$. lipolytica (33) and discussed as protective mechanism against excessive oxygen radical formation (34). Preparations of $Y$. lipolytica complex I are always in the so-called deactive (D) form that slowly reverts into the active (A) form upon addition of substrates (33). Consistent with the very low catalytic activity of the D-form, the structure of $Y$. lipolytica complex I shows the ubiquinone binding-site at markedly greater distance from cluster N2 as compared to the always-active structure of the bacterial enzyme, in which ubiquinone was positioned much closer to its electron-donor, allowing for efficient electron-transfer (10). Supporting this interpretation, loop TMH1-2 ${ }^{\text {ND3 }}$ 
undergoes conformational changes during the A/D-transition (35). Considering the remarkably high sequence conservation within the structural elements involved (Fig. S9) (10, 30) and because binding of ubiquinone next to cluster $\mathrm{N} 2$ is also supported by exhaustive mutagenesis data for the mitochondrial enzyme $(21,27,30,31)$, we propose that in the A-form the ubiquinone binding-pocket of $Y$. lipolytica complex I adopts a conformation similar to the bacterial enzyme that has no D-form. We suggest that the interface region of complex I can switch between two distinct conformational states thereby significantly shifting the ubiquinone binding-site. This switch involves a concerted movement of loops from three subunits (49-kDa, ND1, ND3).

\section{Mechanistic implications}

The central question concerning the mechanism of energy-conversion of complex I is how the redox energy released exclusively in the peripheral arm is transmitted to the proton-pump modules of the membrane arm (Fig. 6). Several lines of evidence indicate that ubiquinone reduction plays a pivotal role in this process. The hypothetical two-state stabilization-change mechanism (36) proposes that stabilization of negatively-charged quinone-intermediates drives a conformational change, thereby transmitting energy to the membrane arm to drive protonpumping. It postulates that complex I switches between the E-state, in which ubiquinone can be reduced by cluster $\mathrm{N} 2$, and the $\mathrm{P}$-state, in which it is moved away from its electron-donor. This description is reminiscent of the two conformational states discussed above. A displacement of the acidic loop TMH5- $6^{\mathrm{ND} 1}$ at the start of the chain of titratable residues reaching through the membrane arm seems ideally suited to transmit an "electrostatic pulse" (37). We therefore hypothesize that an orchestrated movement of the three loops associated with the A/D transition could also reflect the critical energy-converting steps during catalytic turnover (Fig. 6). Such mechanism would imply that the E-state corresponds essentially to the A-form, while the P-state 
would resemble the D-form, with the notable exception that the D-form cannot revert rapidly and spontaneously to the E/A state, because this is prevented by a yet unidentified structural feature not present in the bacterial enzyme. Considering that the A/D-transition is only observed with eukaryotic enzymes, it is tempting to speculate that stabilization of the D-state may involve nearby accessory subunits like NUEM that has been shown to take part in the associated conformational changes (38).

Notably, this series of events could be triggered not only by stabilization of ubisemiquinone, but also of the ubiquinol-anion resulting from the second reduction step (36). While so far experimental evidence for such a second pump-stroke is missing, partitioning of the free energy change by equally involving both electron-transfer steps to ubiquinone seems to make thermodynamic and mechanistic sense, in particular considering that complex I can operate in reverse as a proton gradient driven $\mathrm{NAD}^{+}$reductase.

The described mechanistic principle - charge-induced conformational changes that result in secondary electrostatic polarization of charged residues - may also be important to drive the individual proton-pumping sites of complex I. Defined localized conformational changes should ensure controlled vectorial charge-translocation, while energy-transfer between the sites could occur by electrostatic coupling. Indeed, recent large-scale molecular dynamics simulations suggested that long-range energy transmission in complex I is executed through charge induced protonation-changes of key residues (39).

\section{REFERENCES AND NOTES}

1. U. Brandt, Annu Rev Biochem 75, 69-92 (2006).

2. J. Hirst, Annu Rev Biochem 82, 551-575 (2013).

3. W. J. Koopman, P. H. Willems, J. A. Smeitink, N Engl J Med 366, 1132-1141 (2012). 
4. $\quad$ M. E. Breuer et al., Neurobiol Dis 51, 27-34 (2013).

5. S. Dröse, U. Brandt, Adv Exp Med Biol 748, 145-169.

6. R. Betarbet et al., Nat Neurosci 3, 1301-1306 (2000).

7. S. Kerscher, S. Dröse, K. Zwicker, V. Zickermann, U. Brandt, Biochim Biophys Acta 1555, 83-91 (2002).

8. C. Hunte, V. Zickermann, U. Brandt, Science 329, 448-451 (2010).

9. R. G. Efremov, R. Baradaran, L. A. Sazanov, Nature 465, 441-445 (2010).

10. R. Baradaran, J. M. Berrisford, G. S. Minhas, L. A. Sazanov, Nature 494, 443-448 (2013).

11. R. G. Efremov, L. A. Sazanov, Nature 476, 414-420 (2011).

12. C. C. Page, C. C. Moser, X. Chen, P. L. Dutton, Nature 402, 47-52 (1999).

13. C. Hunte et al., Nature 435, 1197-1202 (2005).

14. E. Screpanti, C. Hunte, J Struct Biol 159, 261-267 (2007).

15. N. J. Hu, S. Iwata, A. D. Cameron, D. Drew, Nature 478, 408-411 (2011).

16. V. K. Moparthi et al., Biochim Biophys Acta 1837, 178-185 (2014).

17. K. R. Vinothkumar, J. Zhu, J. Hirst, Nature, doi:10.1038/nature13686, published online 07 September 2014 (2014).

18. B. C. Marreiros, A. P. Batista, A. M. Duarte, M. M. Pereira, Biochim Biophys Acta 1827, 198-209 (2013).

19. R. G. Efremov, L. A. Sazanov, Nature 476, 414-U462 (2011).

20. A. Galkin, S. Dröse, U. Brandt, Biochim Biophys Acta 1757, 1575-1581 (2006).

21. N. Kashani-Poor, M. Zwicker, S. Kerscher, U. Brandt, J Biol Chem 276, 24082-24087 (2001).

22. H. Jörnvall et al., Biochemistry 34, 6003-6013 (1995).

23. A. Abdrakhmanova, K. Zwicker, S. Kerscher, V. Zickermann, U. Brandt, Biochim Biophys Acta 1757, 1676-1682 (2006). 
24. M. Ciano, M. Fuszard, H. Heide, C. H. Botting, A. Galkin, FEBS Lett 587, 867-872 (2013).

25. V. Zickermann et al., J Biol Chem 278, 29072-29078 (2003).

26. V. Zickermann, B. Barquera, M. Wikström, M. Finel, Biochemistry 37, 11792-11796 (1998).

27. H. Angerer et al., Biochim Biophys Acta 1817, 1776-1784 (2012).

28. J. G. Okun, P. Lümmen, U. Brandt, J Biol Chem 274, 2625-2630 (1999).

29. M. A. Tocilescu et al., Biochim Biophys Acta 1797, 625-632 (2010).

30. M. A. Tocilescu, U. Fendel, K. Zwicker, S. Kerscher, U. Brandt, J Biol Chem 282, 2951429520 (2007).

31. U. Fendel, M. A. Tocilescu, S. Kerscher, U. Brandt, Biochim Biophys Acta 1777, 660-665 (2008).

32. A. B. Kotlyar, A. D. Vinogradov, Biochim Biophys Acta 1019, 151-158 (1990).

33. E. Maklashina, A. B. Kotlyar, G. Cecchini, Biochim Biophys Acta 1606, 95-103 (2003).

34. E. T. Chouchani et al., Nat Med 19, 753-759 (2013).

35. A. Galkin et al., J Biol Chem 283, 20907-20913 (2008).

36. U. Brandt, Biochim Biophys Acta 1807, 1364-1369 (2011).

37. L. Euro, G. Belevich, M. I. Verkhovsky, M. Wikström, M. Verkhovskaya, Biochim Biophys Acta 1777, 1166-1172 (2008).

38. M. Babot et al., Biochim Biophys Acta 1837, 929-939 (2014).

39. V. R. Kaila, M. Wikström, G. Hummer, Proc Natl Acad Sci U S A 111, 6988-6993 (2014).

40. W. Kabsch, Acta Crystallogr D Biol Crystallogr 66, 125 (2010).

41. T. G. Battye, L. Kontogiannis, O. Johnson, H. R. Powell, A. G. Leslie, Acta Crystallogr D Biol Crystallogr 67, 271 (2011).

42. P. R. Evans, G. N. Murshudov, Acta Crystallogr D Biol Crystallogr 69, 1204 (2013). 
43. G. Bricogne, C. Vonrhein, C. Flensburg, M. Schiltz, W. Paciorek, Acta Crystallogr D Biol Crystallogr 59, 2023 (2003).

44. K. Cowtan, Newsletter on Protein Crystallography 31, 34-38 (1994).

45. M. D. Winn et al., Acta Crystallogr D Biol Crystallogr 67, 235 (A2011).

46. P. Emsley, B. Lohkamp, W. G. Scott, K. Cowtan, Acta Crystallogr D Biol Crystallogr 66, 486 (2010).

47. P. D. Adams et al., Acta Crystallogr D Biol Crystallogr 66, 213 (2010).

48. J. Soding, A. Biegert, A. N. Lupas, Nucleic Acids Res 33, W244 (2005).

49. G. Bricogne et al., BUSTER version 2.10.0, Cambridge, United Kingdom: Global Phasing Ltd. (2011).

50. O. S. Smart et al., Acta Crystallogr D Biol Crystallogr 68, 368 (2012).

51. M. Strong et al., Toward the structural genomics of complexes: crystal structure of a PE/PPE protein complex from Mycobacterium tuberculosis. Proc Natl Acad Sci U S A 103, 8060-8065 (2006).

52. V. B. Chen et al., Acta Crystallogr D Biol Crystallogr 66, 12 (2010).

53. E. Chovancová et al., PLoS Computational Biology 8, e1002708, (2012).

54. R. Djafarzadeh et al., Biochim Biophys Acta 1459, 230 (2000).

55. H. Lehmann, L. LaVecchia, Org Process Res Dev 14, 650 (2010).

56. L. Holm, C. Sander, Science 273, 595-603 (1996).

57. K. L. Kavanagh, H. Jornvall, B. Persson, U. Oppermann, Cell Mol Life Sci 65, 3895 (2008).

58. C. Rosano et al., J Mol Biol 303, 77 (2000).

\section{Acknowledgements:}

This work was supported by the German Research Foundation (CRC 746 to C.H.; ZI 552/3-1 to V.Z.) and the Excellence Initiative of the German Federal and State Governments (EXC 115 to 
H.S., U.B. and V.Z; EXC 294 BIOSS to C.H.). The authors thank SLS and ESRF for beamline access and staff support during visits. Excellent technical assistance by Andrea Duchene and Gudrun Beyer is gratefully acknowledged. 


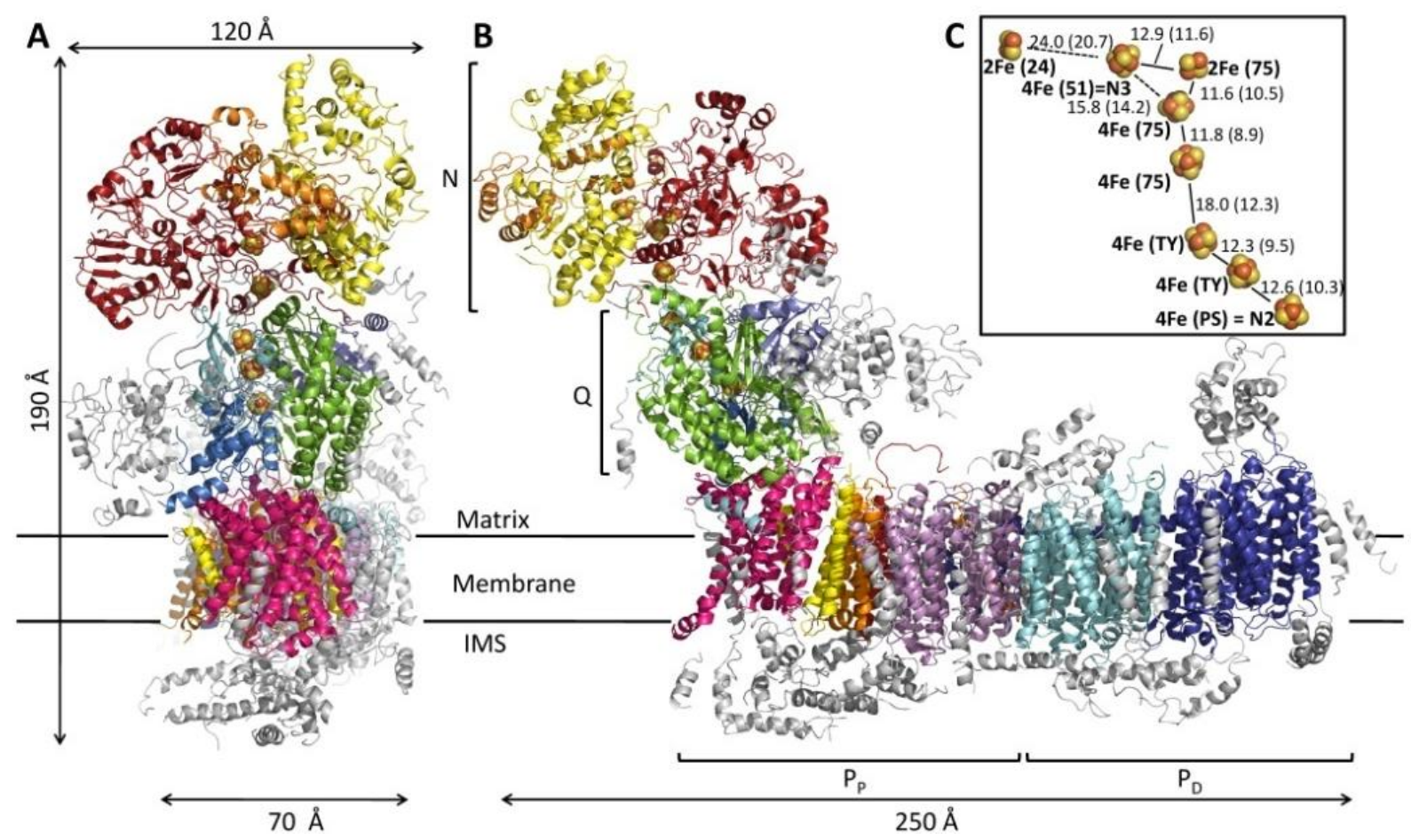

Figure 1 Overall structure. View from peripheral arm (A) and rotated $90^{\circ}(\mathbf{B})$. N-module: 75 kDa/red, 51-kDa/yellow, 24-kDa/orange; Q-module: 49-kDa/green, 30-kDa/violet, PSST/blue, TYKY/cyan; $\mathrm{P}_{\mathrm{P}}$-module: ND5/dark blue, ND4/cyan; $\mathrm{P}_{\mathrm{D}}$-module: ND2/pink, ND4L/red, ND6/orange, ND3/yellow, ND1/pink. Accessory subunits depicted in grey. C, Arrangement of $4 \mathrm{Fe}-4 \mathrm{~S}(4 \mathrm{Fe})-$ and $2 \mathrm{Fe}-2 \mathrm{~S}(2 \mathrm{Fe})$-clusters in the peripheral arm. The coordinating subunits are referred to in brackets. Center-to-center and edge-to-edge (in brackets) distances are in $\AA$. 


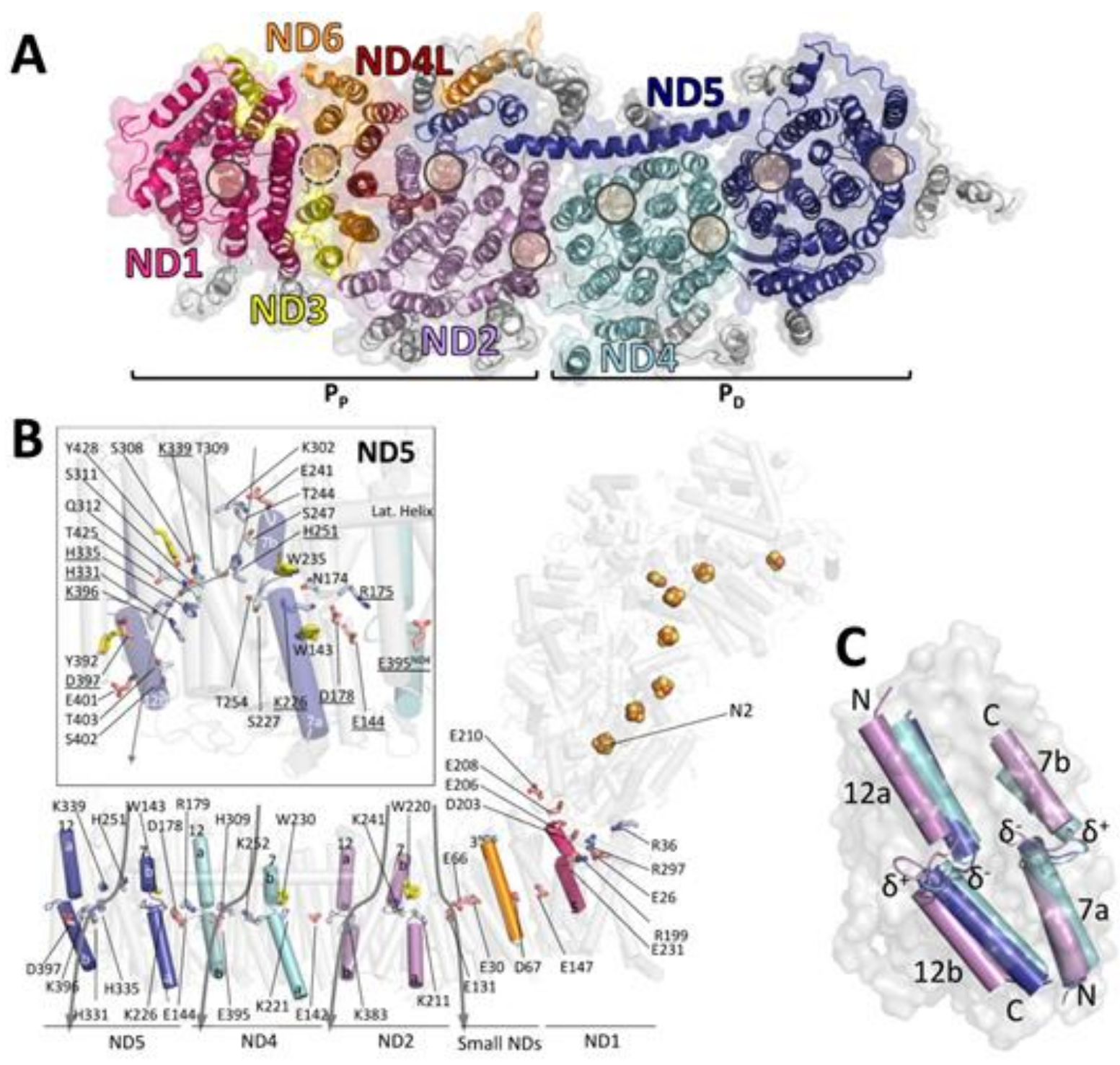

Figure 2 Membrane arm. A, central transmembrane subunits and TMHs of accessory subunits seen from matrix-side (colors as Fig. 1). Solid circles, discontinuous helices in ND1, ND2, ND4 and ND5; broken circle, TMH3 ${ }^{\mathrm{ND} 6}$ containing a $\pi$-bulge. $\mathbf{B}$, discontinous helices and side-chains of residues of the central axis. Insert, arrays of polar and titratable residues of ND5. C, Discontinuous TMH7 and TMH12 after global superposition of ND2, ND4 and ND5 seen along the long axis of the membrane arm. $\delta^{+}, \delta^{-}$, partial charges imposed by helix dipoles. 


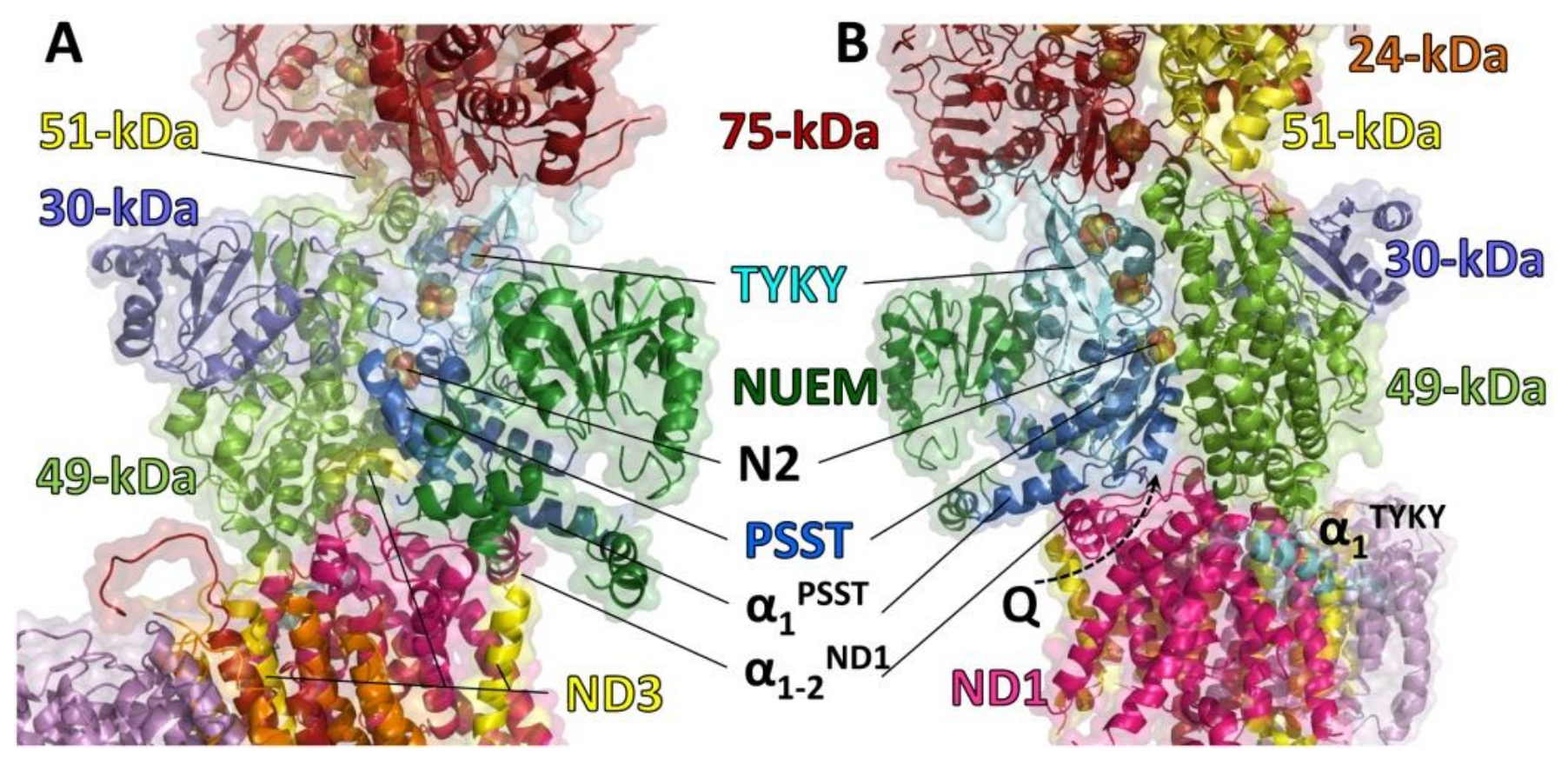

Figure 3 Interface between peripheral and membrane arm. Two views of the interface between central subunits of the Q-module and the $\mathrm{P}_{\mathrm{P}}$-module (colors as Fig. 1) and accessory subunit NUEM (dark green). Dashed arrow, putative access for ubiquinone. 

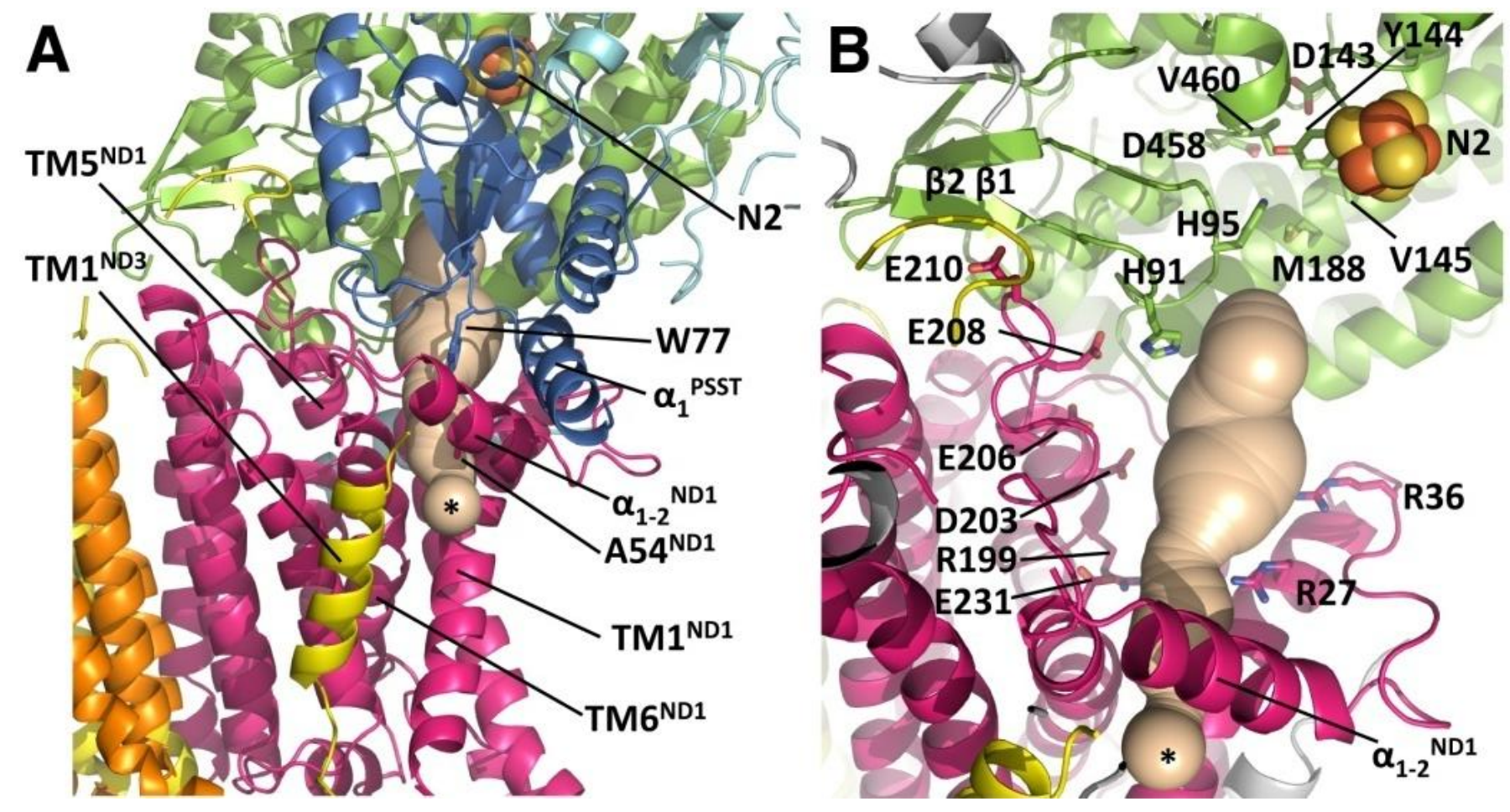

Figure 4 Access path of quinone to the active site. A, side-view. An about $30 \AA$ long cavity (beige) connects the active site below cluster N2 with the matrix bilayer leaflet permitting substrate access. A constricted opening (*) is located between the V-shaped arrangement of TMH1 and TMH6 and below the amphipathic helix $\alpha_{1-2}{ }^{\text {ND1 }}$ (colors as Fig. 1). B, View from PSST, which has been removed for clarity. 


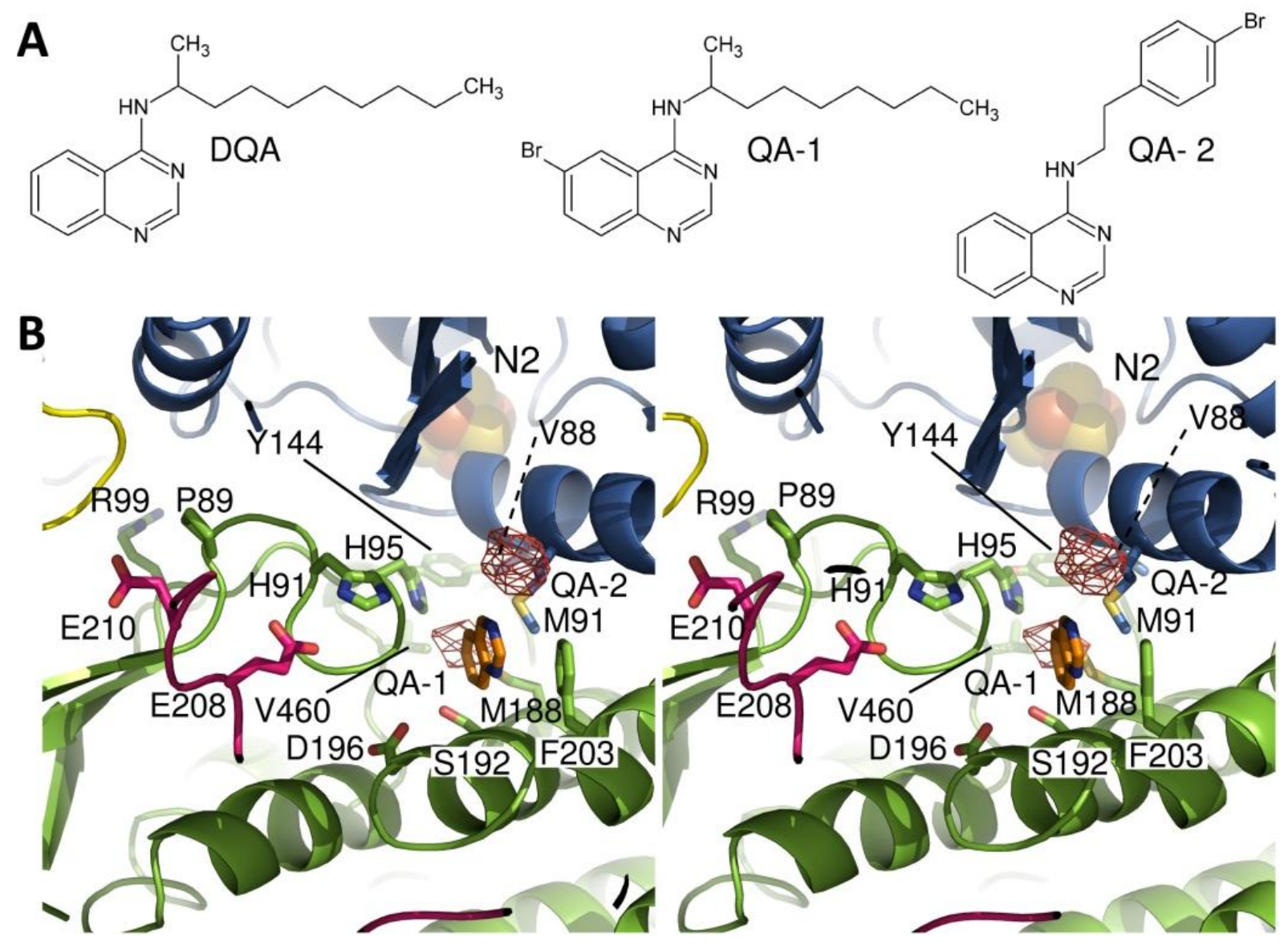

Figure 5 Binding site of the ubiquinone analogous inhibitor DQA. A, constitutions of DQA and bromo-substituted derivatives QA-1 and QA-2. B, stereo-view of inhibitor binding pocket (colors as Fig 1). Single peaks in the bromine anomalous Fourier electron-density maps are

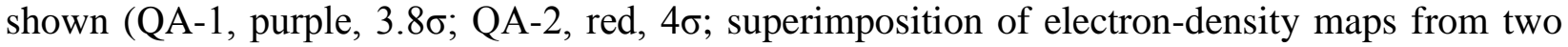
separate experiments on structure). Orange, quinazoline ring modeled into the site. 


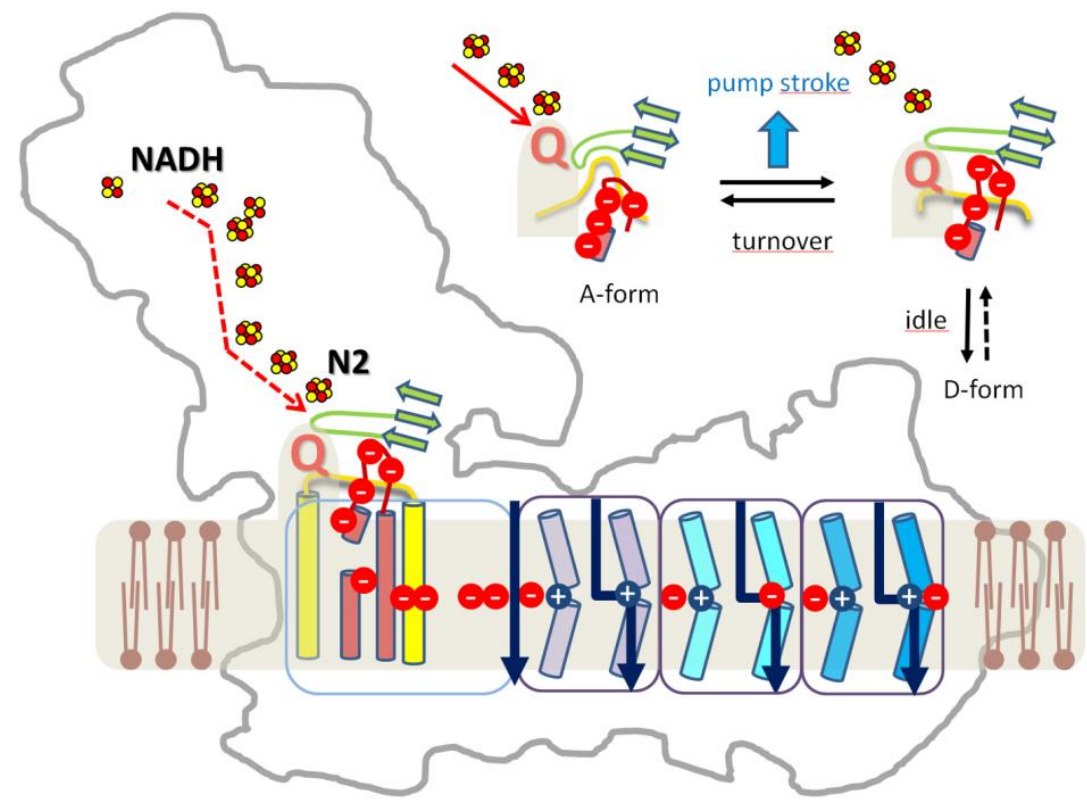

Figure 6 Hypothetical two-state stabilization change mechanism. Electrons are transferred (red arrow) via a chain of iron-sulfur clusters from NADH to ubiquinone (Q). Within three antiporter like subunits (violet frame) and the other ND-subunits, a pattern of titratable residues defines a central axis in the membrane connected to the IMS and matrix-side by putative protontranslocation-pathways (dark blue). Loop TMH5-6 ${ }^{\mathrm{ND} 1}$ (red); loop $\beta_{1}-\beta_{2}{ }^{49-\mathrm{kDa}}$ (green) and the tip of loop TMH1-2 ${ }^{\mathrm{ND} 3}$ (yellow) line the ubiquinone exchange-cavity. During turnover these loops perform a coordinated rearrangement resulting in a shift of the ubiquinone binding site and movement of the cluster of negative charges in loop TMH5- $6^{\mathrm{ND} 1}$, which may trigger an electrostatic pulse towards the membrane arm. Stabilization of the anionic species in the site leads to transition from E-state (left) to P-state (right) driving a stroke of proton pumping. The idling enzyme can convert reversibly from the active A-form into the deactive D-form with a structure similar to the P-state. 